GUT MOTILITY

\title{
Deranged smooth muscle $\alpha$-actin as a biomarker of intestinal pseudo-obstruction: a controlled multinational case series
}

\author{
C H Knowles, D B A Silk, A Darzi, B Veress, R Feakins, A H Raimundo, T Crompton, E C Browning, \\ G Lindberg, J E Martin
}

Gut 2004;53:1583-1589. doi: 10.1136/gut.2003.037275

\begin{abstract}
See end of article for authors' affiliations

....................

Correspondence to: Professor J E Martin, Institute of Pathology, Stepney Way, Royal London Hospital, Whitechapel, London E1 1BB, UK; j.e.martin@ qmul.ac.uk
\end{abstract}

Revised version received 15 March 2004 Accepted for publication 18 March 2004

\begin{abstract}
Background and aims: Chronic idiopathic intestinal pseudo-obstruction (CIIP) is a severe motility disorder associated with significant morbidity. Several histopathological (neuropathic and myopathic) phenotypes have been described but only a single adult with jejunal smooth (circular) muscle $\alpha$-actin deficiency. We present a prospective multinational case series investigating smooth muscle $\alpha$-actin deficiency as a biomarker of this disease.

Methods: A total of 115 fully clinically and physiologically (including prolonged (24 hour) ambulatory jejunal manometry) characterised CIIP patients from three European centres were studied. Immunohistochemical localisation of actins and other cytoskeletal proteins were performed on laparoscopic full thickness jejunal biopsies and compared with adult controls. Distribution of $\alpha$-actin was also characterised in other gut regions and in the developing human alimentary tract.

Results: Twenty eight of 115 (24\%) CIIP patient biopsies had absent $(n=22)$ or partial $(n=6)$ jejunal smooth muscle $\alpha$-actin immunostaining in the circular muscle layer. In contrast, smooth muscle $\alpha$-actin staining was preserved in the longitudinal muscle and in adult jejunal controls $(n=20)$. Comparative study of other adult alimentary tract regions and fetal small intestine, suggested significant spatial and temporal variations in smooth muscle $\alpha$-actin expression.

Conclusions: The ability to modulate $\alpha$-smooth muscle actin expression, evident in development, is maintained in adult life and may be influenced by disease, rendering it a valuable biomarker even in the absence of other structural abnormalities.
\end{abstract}

$\mathrm{P}$ seudo-obstruction describes a condition in which the bowel fails to propel its contents in the presence of an unobstructed lumen. When this problem predominantly affects the small bowel, the condition is labelled intestinal pseudo-obstruction. ${ }^{1}$ Patients commonly present with severe chronic "obstructive" symptoms (abdominal pain, distension/fullness, nausea/vomiting, and intractable constipation) usually with episodic exacerbations that can lead to multiple laparotomies. ${ }^{12}$ The historical diagnostic requirement for plain or contrast radiographic demonstration of air-fluid levels during acute episodes ${ }^{3}{ }^{4}$ is now often replaced by the combination of typical symptoms with proof of abnormal small bowel motility. ${ }^{5}$

While some intestinal pseudo-obstruction cases occur in association with systemic endocrine and connective tissue diseases or malignancy, many have no recognisable aetiology and are described as idiopathic: chronic idiopathic intestinal pseudo-obstruction (CIIP). ${ }^{6}$ Patients can similarly be divided into relatively rare cases evident from birth in which a genetic, $^{7}$ sometimes hereditary, ${ }^{8}$ aetiology can be implicated, and more common cases arising later in life. Sequalae such as psychiatric disorders, opiate dependence (for severe abdominal pain), and malnutrition with requirement for parenteral nutrition with attendant morbidity and mortality are well recognised. ${ }^{2}{ }^{9}$ Although uncommon, these patients individually thus represent a major burden to healthcare providers.

Two relatively new techniques have revolutionised the investigation of CIIP. Direct measurement of changes in intraluminal pressure by small bowel manometry first described abnormalities of intestinal motility in CIIP in the 1980 s. $^{510}$ A number of characteristic qualitative ${ }^{510-13}$ and quantitative abnormalities ${ }^{14}$ are now well accepted by worldwide working party groups. ${ }^{13} 15$ Solid state technological advances now permit prolonged 24 hour ambulatory recordings. ${ }^{15}$

Historically, the description of structural abnormalities (see below) in CIIP utilised tissue provided by bowel resections. Unfortunately, subsequent experience has now demonstrated that surgery, other than perhaps decompression stomas, is rarely effective in CIIP, and often leads to worsening of symptoms and nutrition. ${ }^{9}$ Regrettably, nearly all such abnormalities lie in the enteric neuronal plexuses or in the muscularis propria and are thus not amenable to examination on endoscopic mucosal biopsies. In this respect, the advance of minimally invasive surgical techniques has permitted the safe performance of laparoscopic intestinal biopsy $^{17}{ }^{18}$ providing adequate full thickness tissue for subsequent detailed light microscopic (routine and immunocytochemical) and ultrastructural analysis.

Numerous neuropathies and myopathies are now described in both congenital and acquired primary and secondary forms of CIIP and include those characterised by various structural abnormalities (for example, degeneration or inflammation, etc). ${ }^{21920}$ Among myopathic phenotypes, a deficiency of one of the isoforms of the cytoskeletal smooth muscle protein actin, $\alpha$-actin, has been described in the circular muscle of the jejunum of a single CIIP patient. ${ }^{21}$ As part of an ongoing prospective detailed study of tissue from

Abbreviations: CIIP, chronic idiopathic intestinal pseudo-obstruction; ICCs, interstitial cells of Cajal 
adults with physiologically proven CIIP from three European tertiary referral centres, we aimed to determine the prevalence of this structural phenotype in patients with CIIP and to determine normal expression of $\alpha$-actin in the developing and adult gastrointestinal tract.

\section{MATERIALS AND METHODS}

The study was approved by the local ethics committee.

\section{Control and fetal tissues}

Gastric $(n=5)$, jejunal $(n=20)$ (median age 60 years, 11 females), terminal ileal $(n=12)$, and colonic $(n=12)$ tissues were obtained from adult patients undergoing open surgery for neoplasia in the absence of obstruction or inflammation (confirmed histologically) to observe the distribution of smooth muscle $\alpha$-actin in the "normal" adult gastrointestinal tract. The distribution of smooth muscle $\alpha$-actin was also examined in the developing gastrointestinal tract using 41 fetal small bowel samples from the three trimesters.

\section{Patients}

CIIP was defined on the basis of $>1$ year history of characteristic symptoms - that is, chronic abdominal pain, distension, nausea, and constipation with a history of episodic acute presentations with clinically suspected obstruction and without recognisable aetiology. Patients all had proven abnormal small bowel motility and/or radiological evidence of dilated loops during acute episodes (some also had chronically dilated small bowel without remission). Patients were derived from those attending three tertiary referral centres for specialist investigation and management (Department of Gastroenterology, Huddinge Institute, Stockholm, Sweden-centre 1; St Barts and the Royal London NHS Trust-centre 2; and the Central Middlesex Hospital, London-centre 3). Over the time period of the prospective study (1987-1998 for centre 1; 1989-2002 for centre 2; and 2000-2002 for centre 3), 115 patients (34 males, 81 females, median age 43 (13) years (range 11-74)) with a final diagnosis of CIIP based on the above criteria were investigated. Interestingly, mean age was almost identical (42 $v 43$ years) and sex ratio similar (26\% v 32\% male) between the Scandinavian and British series.

\section{Prolonged small bowel manometry}

Jejunal motility was monitored via three miniature strain gauge transducers mounted on a soft nasojejunal catheter and located at $15 \mathrm{~cm}$ intervals from the catheter-tip: (1) in the duodenum, (2) at the ligament of Treitz, and (3) in the proximal jejunum. Intraluminal pressure changes were sampled at a frequency of $5 \mathrm{~Hz}$ and transmitted signals stored on a portable digital recorder. Study duration was 24 hours and included diurnal (fasting and postprandial) and nocturnal recordings. Details of intubation methods and

\begin{tabular}{|c|c|c|c|}
\hline \multirow[b]{2}{*}{ Tissue site } & \multirow[b]{2}{*}{$\mathbf{n}$} & \multicolumn{2}{|c|}{ Deficient $\alpha$-actin } \\
\hline & & Complete & Partial \\
\hline \multicolumn{4}{|l|}{ Stomach } \\
\hline Control adult & 5 & 0 & 0 \\
\hline CIIP & 5 & 0 & 0 \\
\hline \multicolumn{4}{|l|}{ Jejunum } \\
\hline Control adult & 20 & 0 & 0 \\
\hline CIIP & 115 & 22 (19\%) & $6(5 \%)^{*}$ \\
\hline \multicolumn{4}{|l|}{ lleum } \\
\hline Control adult & 12 & $2(17 \%)$ & $10(83 \%)$ \\
\hline CIIP & 13 & $7(54 \%)$ & $2(15 \%)$ \\
\hline \multicolumn{4}{|l|}{ Colon } \\
\hline Control adult & 12 & 0 & 0 \\
\hline CIIP & 35 & 0 & 0 \\
\hline \multicolumn{4}{|c|}{ Fetal small intestine } \\
\hline 1st trimester & 2 & 0 & \\
\hline 2nd trimester & 18 & $2(11 \%)$ & \\
\hline 3rd trimester & 21 & $19(91 \%)$ & \\
\hline
\end{tabular}

the study protocol are published elsewhere. ${ }^{22}$ Qualitative analysis examined for a variety of characteristic patterns of abnormality based on previously published criteria. ${ }^{5}{ }^{13}$ Automated semiquantitative data analysis was performed (centres 1 and 2 only) using a validated computer program for identification of contractile events, as described previously, ${ }^{23}$ and abnormalities defined using normal ranges. ${ }^{22}{ }^{24}$

\section{Laparoscopic biopsies}

Extra $^{-18}$ and intracorporeal ${ }^{17}$ methods of antimesenteric full thickness jejunal biopsy were performed depending on surgeon preference without serious complications in any patient. To standardise as much as possible the site of biopsy, and be in maximal accordance with the position of the distal sensor of manometric studies, biopsies were all taken from the most proximal easily accessible loop of jejunum by tracing the bowel forward from the duodenojejunal flexure (usually $15-20 \mathrm{~cm}$ from the ligament of Trietz). Centres 1 and 2 utilised an extracorporeal technique in the most part (that is, laparoscopically assisted) whereas centre 3 performed all biopsies intracorporeally using an endoGIA stapling device (technique not previously described for this purpose: mean operating time 13 minutes). Samples were fixed overnight in $10 \%$ formalin solution and mounted in paraffin blocks by standard methodology. Smaller numbers of gastric, ileal, and colonic biopsies were also performed in some patients.

\section{Histopathology}

All control and CIIP tissues were handled in an identical manner. Sections $(5 \mu \mathrm{m})$ were stained for a panel of routine

Table 1 Immunostains used in this study

\begin{tabular}{|c|c|c|c|c|}
\hline \multirow{2}{*}{$\begin{array}{l}\text { Antibody } \\
\text { Vimentin }\end{array}$} & \multirow{2}{*}{$\begin{array}{l}\text { Clone } \\
\text { (Vim 3B4) }\end{array}$} & \multicolumn{2}{|c|}{ Manufacturer and type } & \multirow{2}{*}{$\begin{array}{l}\text { Retrieval } \\
\text { None }\end{array}$} \\
\hline & & Dako & Monoclonal mouse antihuman & \\
\hline Desmin & (D33) & Dako & Monoclonal mouse antihuman & None \\
\hline $\begin{array}{l}\text { Smooth muscle } \alpha \text {-actin } \\
\text { (specificity against } \alpha \text {-isoactin only) }\end{array}$ & (1A4) & Dako & Monoclonal mouse antihuman & None \\
\hline $\begin{array}{l}\text { Muscle actin } \\
\text { (specificity against } \alpha \text { - and } \gamma \text {-isoactin)) }\end{array}$ & (HHF35) & Dako & Monoclonal mouse antihuman & None \\
\hline $\begin{array}{l}\text { Sarcomeric actin } \\
\text { (specificity against sarcomeric actin only) }\end{array}$ & $(\alpha-S r-1)$ & Dako & Monoclonal mouse antirabbit & None \\
\hline Smooth muscle $\alpha$-actin* & (1A4) & Dako & Monoclonal mouse antihuman & Microwave \\
\hline Smooth muscle $\alpha$-actin* & $(\alpha s m-1)$ & NC & Monoclonal mouse antihuman & None \\
\hline
\end{tabular}

*Five cases with absent $\alpha$-actin staining were confirmed by use of a further commercially available antibody and method of antigen retrieval. 

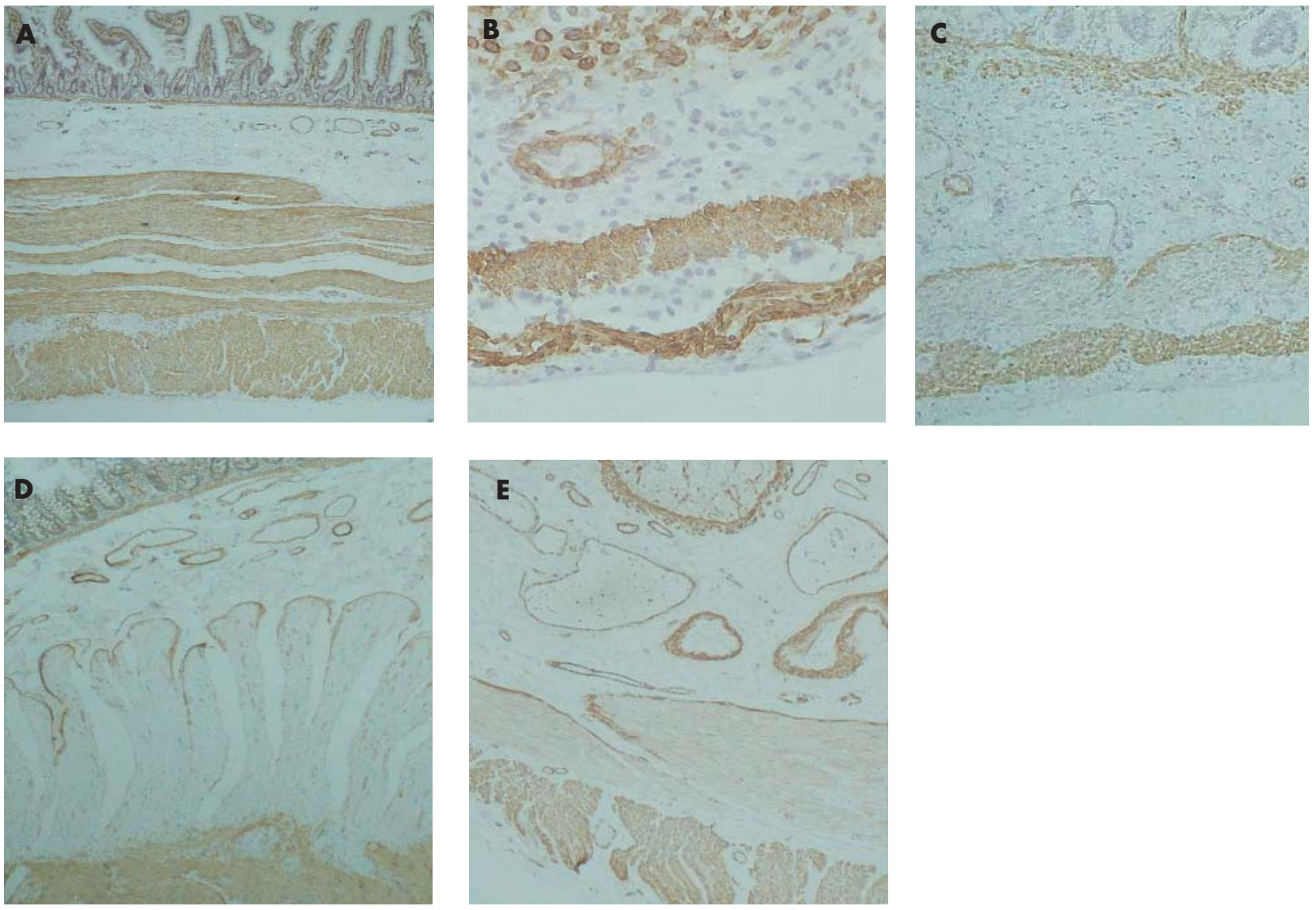

Figure $1 \alpha$-Actin immunostaining of sections of human intestine. (A) Control adult jejunum with a normal pattern of $\alpha$-actin immunostaining in both layers. (B) Fetal ileum (20/21 weeks' gestation) with strong immunostaining in both layers. (C) Fetal ileum (38/39 weeks' gestation) with almost absent immunostaining in the circular muscle layer. (D) Jejunal full thickness laparoscopic biopsy from a patient with chronic idiopathic intestinal pseudoobstruction (CIIP) showing complete deficiency of $\alpha$-actin immunostaining in the circular layer of the muscularis propria but preservation of staining elsewhere. (E) A similar biopsy from a patient with CIIP and partial $\alpha$-actin immunostaining.

(haematoxylin-eosin, periodic acid-Schiff) and immunohistochemical stains. Immunostaining was performed using a kit based (Dako, Ely, Cambs, UK) indirect avidin-biotin peroxidase complex method on transversely and/or parallel cut sections for detection of degenerative and inflammatory, neuropathic, and myopathic abnormalities described in CIIP. ${ }^{219} 20$ The panel of antibodies specifically included for smooth muscle epitopes are shown in table 1. Neural elements were immunostained using antibodies to neurone specific enolase, protein gene product 9.5, S100, and neurofilament $2 \mathrm{~F} 11$. These are fully referenced elsewhere. ${ }^{25}$ A proportion of more recently investigated patients from centre $1 \quad(n=13 / 63)$ also underwent immunostaining for abnormalities in interstitial cells of Cajal (ICC) using c-kit antibody. Appropriate negative (replacement of primary antibodies with pooled immunoglobulin) controls were employed. The presence of staining within other actin expressing elements on each slide (see results) was deemed itself to act as a positive control. In addition, five cases with absent $\alpha$-actin staining were confirmed by the use of a further commercially available antibody and method of antigen retrieval (table 1).

\section{Statistical analysis}

The study is largely descriptive. A single contingency analysis $\left(\chi^{2}\right)$ was performed for the primary end point (proportions of controls $v$ CIIP patients with complete or absent circular muscle $\alpha$-actin deficiency in the jejunum).

\section{RESULTS}

\section{Control tissues (table 2)}

All adult control gastric $(n=5)$, jejunal $(n=20)$ (fig lA), and colonic tissues $(n=12)$ had smooth muscle $\alpha$-actin immunostaining in both muscle layers. In contrast, of 12 ileal control tissues studied, all had absent $(\mathrm{n}=2,17 \%)$ or partially absent $(\mathrm{n}=10,83 \%)$ staining in the circular muscle layer (in the characteristic pattern observed in CIIP patients in the jejunum (see below)). Significant developmental alterations in $\alpha$-actin staining were also observed in the fetal ileum, with strong immunostaining in the majority in earlier development (first and second trimesters: 18/20 (90\%); fig 1B) but then weak or absent staining consistent with the adult pattern in the majority in the third trimester (19/21 (91\%); fig $1 C)$.

\section{CIIP patients (table 2)}

Of 115 biopsies studied from CIIP patients (who all met clinical, manometric, or radiological inclusion criteria), 22 (19\%) had absent jejunal smooth muscle $\alpha$-actin immunostaining in the circular muscle layer with the exception of its innermost layer adjacent to the submucosa where smooth muscle cells stained strongly positive (fig 1D). In six further cases $(5 \%)$, there was incomplete (partial) loss from the circular muscle layer (fig $1 \mathrm{E}) \quad(\mathrm{p}<0.05$ compared with controls). In contrast, smooth muscle $\alpha$-actin immunostaining was normal in longitudinal muscle, the muscularis mucosae, and blood vessel walls in all cases. Immunostaining 
Table 3 Other histopathological abnormalities in 115 patients with chronic idiopathic intestinal pseudoobstruction (CIIP) in comparison with $\alpha$-actin deficiency

\begin{tabular}{|c|c|}
\hline$\alpha$-Actin deficiency & $28(24 \%)$ \\
\hline \multicolumn{2}{|l|}{ Neuropathic } \\
\hline Degenerative neuropathy & $20(17 \%)$ \\
\hline Inflammatory plexitis & $20(17 \%)$ \\
\hline Lymphocytic ganglionitis & $13(11 \%)$ \\
\hline \multicolumn{2}{|l|}{ Myopathic } \\
\hline Degenerative leiomyopathy & $16(14 \%)$ \\
\hline Vacuolar myopathy & $7(6 \%)$ \\
\hline Hollow visceral myopathy & $4(3 \%)$ \\
\hline Inclusion body myopathy & $4(3 \%)$ \\
\hline Inflammatory leiomyositis & $1(1 \%)$ \\
\hline \multicolumn{2}{|l|}{ Interstitial cell abnormalities } \\
\hline Decreased number ICCs & $2(15 \%)^{*}$ \\
\hline
\end{tabular}

for other smooth muscle epitopes, including muscle actin (specific for both $\alpha$ - and $\gamma$-isoforms), in the circular muscle layer was equivalent in intensity to other intestinal muscle layers. Absent or markedly reduced smooth muscle $\alpha$-actin immunostaining was validated in 10 patients using a different commercially available antibody as well as a different method of antigen retrieval.

Our findings, on the basis of routine and immunohistochemical methods for neuropathic, myopathic, and ICC abnormalities in the series as a whole, are shown in table 3. Such histological abnormalities were found in a high proportion of CIIP patients overall (81/115 (70\%)) but were less frequent as concomitant findings in specimens with deranged $\alpha$-actin immunostaining $(9 / 28(30 \%))$ : degenerative neuropathy and degenerative myopathy, decreased ICCs (each one case), myenteric plexitis (two cases), and inclusion bodies (two cases). When subanalysed in terms of criteria used for diagnosis of CIIP (radiological dilatation with or without manometry versus manometry alone), the $\alpha$-actin deficient phenotype was more prevalent in the latter (16/36 $(44 \%)$ v 12/79 (15\%)) with well established neuro-/myopathic phenotypes more common in the dilated bowel group.

In common with controls, a pattern of reduced immunoreactivity predominated in the ileum (9/13 (69\%)) but whereas complete absence of immunostaining was $>$ ? uncommon in controls $(2 / 12)$ this was more common in patients $<$ ?show $=$ to] $(7 / 13 \quad(54 \%))$. Furthermore, unlike controls, four patients had normal immunostaining in both layers. Gastric and colonic tissues all had normal immunostaining in both layers.

\section{Clinical and radiological findings (table 4)}

Median age of CIIP patients with smooth muscle $\alpha$-actin deficiency (partial or complete) was 41 (11-68) years (13 male, 15 female). All patients had characteristic pseudoobstructive symptoms (table 2) which were longstanding (median duration 14 years). All patients had severe abdominal pain which was almost always exacerbated by eating (96\%) and often also nocturnal (71\%). Most also had distension, nausea, and vomiting, and two thirds were severely constipated. Nine patients had dilated small bowel loops on plain or contrast radiography during acute episodes and included the five patients who did not complete small bowel manometry (see below) and the two in whom manometry was normal. No patient had clinical or laboratory evidence of diabetes, connective tissue disease, or malignant disease.

\section{Manometric findings}

Twenty six patients with smooth muscle $\alpha$-actin deficiency had undergone prolonged ambulatory small bowel

Table 5 Small bowel manometric abnormalities in 28 chronic idiopathic intestinal pseudo-obstruction patients with smooth muscle $\alpha$-actin deficiency who underwent successful prolonged ( 24 hour) jejunal manometry

\begin{tabular}{ll}
\hline & No of patients (\%) \\
\hline Qualitative abnormalities & $3(13 \%)$ \\
Generalised hypomotility & $17(74 \%)$ \\
Aberrant PIII configuration & $17(74 \%)$ \\
Abnormal PIII propagation & $14(61 \%)$ \\
Bursts of non-propagated activity & $14(61 \%)$ \\
Sustained uncoordinated phasic activity & $12(52 \%)$ \\
Discrete clustered contractions & $8(35 \%)$ \\
Quantitative abnormalities & $7(30 \%)$ \\
Reduced PIII velocity & \\
Reduced or absent MMC $†$ & \\
\hline PIII, phase III. & \\
*Defined as $<1 \mathrm{~cm} /$ minute (Scott and colleagues $\left.{ }^{22}\right)$. \\
†Defined as $\leqslant 2$ migrating motor complexes (MMCs)/24 hours (Scott \\
and colleagues ${ }^{22}$ ).
\end{tabular}

Table 4 Clinical data of 28 patients with smooth muscle $\alpha$-actin deficiency

Symptoms
Duration (y) (median (range))
Abdominal pain
Distension
Nausea/vomiting
Constipation
Diarrhoea
Weight loss
History
Radiological obstructive episodes
Previous laparotomies*
Psychiatric history
Drug history†
Opiates
Antispasmodics
Prokinetics
Laxatives

$14(4-33)$

$28(100 \%)$

$23(82 \%)$

$22(79 \%)$

$17(61 \%)$

$6(21 \%)$

$10(36 \%)$ (6 severe PCM; 5 TPN or HPN)

$9(32 \%)$

$8(29 \%)$ (median 2 (range 1-3))

$6(21 \%)$ (4 depression)

$7(25 \%)$ (5 at time of investigation)

$13(46 \%)$

$13(46 \%)$

$8(29 \%)$

*For obstruction. Further patients underwent laparotomy for related (three subtotal colectomies) and unrelated causes.

tOther drugs utilised with lower frequency included octreotide, proton pump inhibitors, antidiarrhoeals, and steroids.

PCM, protein calorie malnutrition; TPN, total parenteral nutrition; HPN, home parenteral nutrition. 
manometry, the remaining two being too unwell to undergo the investigation. A further three patients failed jejunal intubation, in two cases due to failure of catheter migration beyond the duodenum (itself said to be indicative of propulsive disorder). ${ }^{26}$ Of the 23 completed studies, 21 patients had qualitative and/or quantitative evidence of intestinal pseudo-obstruction based on recognised abnormal patterns of contraction and normal ranges, respectively. The main qualitative and quantitative findings are shown in table 5 and were those generally considered predictive of enteric neuropathy as the dominant lesion. ${ }^{513151622}$ Qualitative abnormalities occurred with similar frequencies to those previously published..$^{5}$ There were no differences in clinical and manometric findings between patients with partial or complete smooth muscle $\alpha$-actin deficiency.

\section{DISCUSSION}

Chronic idiopathic intestinal pseudo-obstruction (CIIP) is a relatively rare but severe disorder whose diagnosis and management are highly problematic. The series presented is to our knowledge the largest published to date, comprising 115 patients from three European centres. In addition, we have used contemporary methodology (small bowel manometry and laparoscopic biopsy) to further study the pathophysiology of each patient in detail. This study has demonstrated that approximately one quarter $(n=28)$ of patients with CIIP have absent or reduced $\alpha$-actin staining in the circular muscle of the jejunum, usually in the absence of other significant structural abnormalities of muscle and nerve, and that this finding is not observed in controls.

It is now over 10 years since a single case of deficient jejunal circular muscle smooth muscle $\alpha$-actin was described in a biopsy from a 48 year old female with clinical, radiological, and physiological features of CIIP but no structural abnormality of muscle or nerve on light or ultrastructural analysis. ${ }^{21}$ The authors concluded, on the basis of the absence of this phenotype in the jejunum of nine other patients with intestinal dysmotility syndromes and three autopsy specimens from infants without gastrointestinal disease, that this represented a specific biochemical phenotype related to the patient's clinical disorder, and which might (principally on the basis of the early onset of symptoms in this patient) be developmental. To our knowledge, only two such further CIIP patients with deficient $\alpha$-actin have subsequently been reported in the literature to date: a single case report of a two year old with inflammatory myositis ${ }^{27}$ and one adult of an uncontrolled series of 11 where the site of small bowel biopsy was not stated and other gross structural myopathic abnormalities were also present..$^{28}$

The significance of the findings made in this study merits full discussion. It is conceivable that the findings relate to technical or methodological errors. However, the biopsies were processed and examined blind to the diagnosis by two observers. Each slide is internally controlled for effectiveness of $\alpha$-actin immunostaining by the presence of immunoreactivity in other smooth muscular structures on the slidethat is, blood vessels, the longitudinal muscle layer, and the innermost layer of the circular muscle (strongly stained in all cases). In addition, cases of absent staining were confirmed by the use of a further commercially available antibody. Furthermore, the abnormalities observed appear to be specific for $\alpha$-actin rather than other muscle markers which all stained normally. Without further investigation however, it is impossible to say whether the protein is absent, hidden, or not staining due to a conformational change.

In smooth muscle contraction, there is an interaction between thick (myosin) and thin (actin) filaments. Actin exists in multiple isoforms (isoactins) which are differentially expressed in a tissue dependent manner. The major isoactins in mature intestinal smooth muscle are $\gamma$ and $\alpha$, the $\gamma$-isoform being the largest constituent and the $\alpha$-form being the smaller yet still important component. ${ }^{29}$ Remarkably little is known of the spatial expression of isoactins along the human alimentary tract in adulthood or of the temporal expression in development. In contrast, both have been well studied by McHugh and colleagues in experimental animals. In adult small mammals, heterogeneous isoactin gene expression has been clearly demonstrated along the length of the gastrointestinal tract. ${ }^{30} 31$ These studies demonstrated a high degree of concordance $(>90 \%)$ between isoactin gene expression and protein production and it is thus assumed that distinct patterns of differential expression are primarily regulated at a transcription level. That similar spatial alterations in isoactin expression also occur in humans in normality is supported by the findings here (albeit only by differences in protein immunostaining), with control gastric, jejunal, and colonic circular muscle $\alpha$-actin immunostaining much stronger than in the ileum. While interpretation of these findings would be supported by further study (western/ northern blotting), there is at least reasonable homology between our findings in humans and those in the rat in this respect, the rat ileum having the lowest relative $\alpha$-actin expression (by a considerable factor) compared with other alimentary tract segments. ${ }^{30}$ Alterations of differential $\alpha$-actin expression in disease, in addition to cases of CIIP described, have also been demonstrated in Hirschsprung's disease ${ }^{32}$ and murine transgenic models thereof, ${ }^{33}$ jejunal atresia, ${ }^{34}$ and in inflammation (discussed below).

In gastrointestinal smooth muscle development of experimental animals, molecular studies have helped redefine the cellular phenotypes involved. ${ }^{35}{ }^{36}$ Undifferentiated mesenchymal cells associated with the gut primordium initially differentiate into a $\alpha$-smooth muscle actin positive and $\gamma$-smooth muscle actin negative cellular phenotype (the myoblast). As development proceeds, the myoblast differentiates into an immature smooth muscle myocyte and thence to a mature myocyte (which corresponds to the classically defined spindle shaped mature smooth muscle cell). Both of these cellular phenotypes coexpress both $\alpha$ - and $\gamma$-isoactins. However, distinct ratios of these contractile proteins are found in each, with predominantly $\alpha$-isoactin expression in the immature myocyte and $\gamma$-isoactin in the mature myocyte in rodent development. That similar temporal alterations of isoactin coexpression ratios occur in humans is supported by our findings of decreasing proportions of ileal tissues with strong $\alpha$-isoactin immunostaining with advancing fetal gestation.

Unlike mature skeletal and cardiac myocytes, mature smooth muscle cells retain the developmental potential to modulate their cellular phenotypes both in vivo and in vitro and this potential remains present in adult visceral tissues. At present, it is not known whether all mature smooth muscle myocytes retain this potential but it is known that a significant proportion never terminally differentiate, so retaining the potential to modulate their cellular phenotype back to a smooth muscle myoblast or indeed differentiate towards myofibroblasts. ${ }^{37}$ Therefore, gastrointestinal smooth muscle cells might be considered to exist on a flexible differentiation line that has at its extremes the smooth muscle myoblast and mature smooth muscle myocyte. Such cellular plasticity thus provides a further potential mechanism by which alterations in differential isoactin expression might be explained in human disease, including CIIP. The relative contribution however of cellular plasticity per se in comparison with regulation of gene expression in a "stable" mature myocyte population have not been studied in human health or disease and would require complex methodology akin to that employed in murine cell culture. ${ }^{36}$ 
The functional significance of the finding of spatial alterations in $\alpha$-smooth muscle actin staining in the context of disease is not immediately clear. Alterations in contractile proteins in disease might be expected to have an effect on motility although the effects of a change in smooth muscle $\alpha$-actin alone (that is, single biochemical abnormality) might be expected to be less profound than that of other significant cytoskeletal or structural abnormalities. However, differential expression of $\alpha$-isoactin in functionally distinct organs (for example, oesophagus versus small bowel, sphincteric versus non-sphincteric tissues ${ }^{31}$ ) of experimental animals supports a functional (that is, pathophysiological) effect of such changes. The disturbances observed in CIIP on prolonged manometry both in the majority of patients in this study and others (predominantly of the MMC with or without abnormal additional non-propulsive activities) are usually said to be indicative of a motor programming abnormality (that is, that caused by neural dysfunction) rather than an intrinsic myopathic disturbance in which a pattern of generalised hypomotility is often observed, ${ }^{13}$ but this remains contentious. Of further difficulty to explain in both this study and others ${ }^{21} 2728$ is the specificity of this finding to the circular muscle layer only. Smith and colleagues ${ }^{21}$ postulated that $\alpha$-actin deficiency might occur as a developmental abnormality confined to this layer because of embryological differences in development between the origin of the two layers. It is hard to see how this could be the case in our series where nearly all cases had a history suggestive of an acquired phenomenon. Whether such changes reflect a difference in function of the two muscle layers can only be the subject of speculation.

The mechanisms leading to altered $\alpha$-isoactin expression in CIIP have not been studied here. Several studies have however demonstrated varying degrees of inflammation both of the muscularis propria itself ${ }^{192027}$ and the myenteric plexus $^{19} 203839$ in patients with CIIP. While overt evidence of inflammation was only present in $2 / 31$ patients with $\alpha$-actin deficiency in this study, there is evidence in rats that actin isoform expression can be modulated by experimental induction of mild inflammation even limited to the mucosa, ${ }^{40}$ and that such immunomodulation of enteric muscle function can persist after the initial stimulus is removed. ${ }^{41}$ The latter observation is supported by a single CIPO case report, described longitudinally, in which progressive loss of $\alpha$-actin immunostaining was demonstrated at three time points despite improvement of the initial florid myositis. ${ }^{27}$

It is our opinion, based on the findings of this study and the diversity of disorders in which abnormalities of isoactin expression have been observed, that the alteration in staining in jejunal circular muscle in CIIP patients, while probably not itself causative or specific to the disorder, at least represents a useful biomarker, especially in the absence of other structural abnormalities. The study also emphasises the importance of precisely defining the location of tissues studied and ages of patients (and controls)/experimental animals in studies where conclusions are drawn regarding histopathological phenotypes in the gastrointestinal tract.

\section{ACKNOWLEDGEMENTS}

We thank Dr I Scheimberg for help with collation of fetal samples and Professor David Wingate for help with the qualitative analysis of the small bowel manometric recordings.

JEM is supported by the Motor Neuron Disease Association, the Medical Research Council, the Annie Lindsell Charitable Trust, and the Birth Defects Foundation.

\section{Authors' affiliations}

C H Knowles, R Feakins, T Crompton, E C Browning, J E Martin, Institute of Cellular and Molecular Science, Barts and the London, Queen Mary's School of Medicine and Dentistry, London, UK
D B A Silk, A H Raimundo, Department of Gastroenterology and Nutrition, Central Middlesex Hospital, London, UK

A Darzi, Department of Surgery, St Mary's University Hospital, London, UK

B Veress, Department of Pathology, University Hospital MAS, Malmö, Sweden

G Lindberg, Department of Medicine, Karolinska Institutet, Huddinge University Hospital, Stockholm, Sweden

\section{REFERENCES}

1 Dudley HAF, Sinclair ISR, McLaren IF, et al. Intestinal pseudo-obstruction. $J R$ Coll Surg Edin 1958:3:206-17.

2 Mann SD, Debinski HS, Kamm MA. Clinical characteristics of chronic idiopathic intestinal pseudo-obstruction in adults. Gut 1997;41:675-81.

3 Rudolph CD, Hyman PE, Altschuler SM, et al. Diagnosis and treatment of chronic intestinal pseudo-obstruction in children. J Pediatr Gastroenterol Nutr 1997;24:102-12

4 Di Lorenzo C. Psudo-obstruction: Current approaches. Gastroenterology 1999;116:980-7.

5 Stanghellini V, Camilleri M, Malagelada JR. Chronic idiopathic intestinal pseudo-obstruction: clinical and intestinal manometric findings. Gut 1987;28:5-12

6 Maldonado JE, Gregg JA, Green PA, et al. Chronic intestinal pseudoobstruction. Am J Med 1970;49:203-12.

7 Auricchio A, Brancolini V, Casari G, et al. The locus for a novel syndromic form of neuronal intestinal pseudoobstruction maps to Xq28. Am J Hum Genet 1996;58:743-8.

8 Rodrigues CA, Shepherd NA, Lennard-Jones JE, et al. Familial visceral myopathy: a family with at least 6 involved members. Gut 1989;30:1285-92.

9 Heneyke S, Smith VV, Spitz L, et al. Chronic intestinal pseudo-obstruction: treatment and long term follow up of 44 patients. Arch Dis Child 1999;81:21-7.

10 Summers RW, Anuras S, Green J. Jejunal manometry patterns in health, partial intestinal obstruction and pseudoobstruction. Gastroenterology 1980;85:1290-300

11 Greydanus MP, Camilleri M. Abnormal postcibal antral and small bowel motility due to neuropathy or myopathy in systemic sclerosis. Gastroenterology 1989;96:110-15

12 Weston S, Thumshirn M, Wiste J, et al. Clinical and upper gastrointestinal motility features in systemic sclerosis and related disorders. Am J Gastroenterol 1998;93:1085-9.

13 Camilleri M, Haslam WL, Parkman HP, et al. Measurement of gastrointestinal motility in the GI laboratory. Gastroenterology 1998;1 15:747-62.

14 Husebye $\mathbf{E}$. The patterns of small bowel motility: physiology and implications in organic disease and functional disorders. Neurogastroenterol Motil 1999:11:141-61.

15 Quigley EM, Deprez PH, Hellstrom P, et al. Ambulatory intestinal manometry: a consensus report on its clinical role. Dig Dis Sci 1997;42:2395-400.

16 Wingate $D$, Hongo $M$, Kellow J, et al. Disorders of gastrointestinal motility: towards a new classification. J Gastroenterol Hepatol 2002;17(suppl):S1-14.

17 Greig JD, Miles WF, Nixon SJ. Laparoscopic technique for small bowel biopsy. Br J Surg 1995;82:363.

18 Tornblom $\mathbf{H}$, Lindberg $G$, Nyberg $B$, et al. Full-thickness biopsy of the jejunum reveals inflammation and enteric neuropathy in irritable bowel syndrome. Gastroenterology 2002;123:2144-7.

19 Smith VV, Milla PJ. Histological phenotypes of enteric smooth muscle disease causing functional intestinal obstruction in childhood. Histopathology 1997:31:112-22.

20 Martin JE, Smith VV, Domizio P. Myopathies of the gastro-intestinal tract. In: Lowe J, Underwood JC, eds. Recent advances in histopathology 18. Edinburgh: Churchill Livingstone, 1999:43-62.

21 Smith VV, Lake BD, Kamm MA, et al. Intestinal pseudo-obstruction with deficient smooth muscle alpha-actin. Histopathology 1992;21:535-42.

22 Scott SM, Picon L, Knowles CH, et al. Automated quantitative analysis of nocturnal jejunal motor activity identifies abnormalities in individuals and subgroups of patients with slow transit constipation. Am J Gastroenterol 2003:98:1123-34.

23 Benson RJ, Castillo FD, Wingate DL, et al. The computer as referee in the analysis of human small bowel motility. Am J Physiol 1993;4:G645-54.

24 Husebye E, Skar V, Aalan OO, et al. Digital ambulatory manometry of the small intestine in healthy adults. Estimates of variation within and between individuals and statistical management of incomplete MMC periods. Dig Dis Sci 1990;35: 1057-65.

25 Knowles CH, Nickols CD, Scott SM, et al. Secondary smooth muscle degeneration with inclusion bodies in slow transit constipation. J Patho $2001 ; 193: 390-7$.

26 Kellow JE. Manometry. In: Schuster MM, Crowell MD, Koch KL, eds. Schuster atlas of gastrointestinal motility in health and disease, 2 nd edn. Hamilton: BC Decker Inc, 2002:219-36.

27 Ruuska TH, Karikoski R, Smith WV, et al. Acquired myopathic intestinal pseudo-obstruction may be due to autoimmune enteric leiomyositis. Gastroenterology 2002;122:1133-9.

28 Moore SW, Schneider JW, Kaschula ROC. Unusual variations of gastrointestinal smooth muscle abnormalities associated with chronic intestinal pseudo-obstruction. Pediatr Surg Int 2002;18:13-20.

29 Fatigati V, Murphy RA. Actin and tropomyosin variants in smooth muscles. Dependence on tissue type. J Biol Chem 1984;259:14383-8. 
30 Liddell RA, Syms M, McHugh KM. Heterogeneous isoactin gene expression in the adult rat gastrointestinal tract. Gastroenterology 1993;105:347-56

31 Liddell RA, Mooers SU, Siegman MJ, et al. Differential isoactin gene expression in the sphincteric and nonsphincteric gastrointestinal smooth muscles of the opossum. Proc Soc Exp Biol Med 1994;205:321-6.

32 Fromont-Hankard G, Lafer D, Masood S. Altered expression of alpha-smooth muscle actin in Hirschsprung's disease. Arch Pathol Lab Med 1996; 120:270-4

33 Liddell RA, Mooers SU, Siegman MJ, et al. Altered isoactin gene expression in the affected bowel segments of the lethal spotted mouse. Gastroenterology 1993; 105:441-8

34 Masumoto K, Suita S, Nada O, et al. Abnormalities of enteric neurons, intestinal pacemaker cell and smooth muscle in human intestinal atresia. J Pediatr Surg 1999:34:1463-8.

35 McHugh, KM. Molecular analysis of gastrointestinal smooth muscle development. J Pediatr Gastroenterol Nutr 1996;23:379-94.
36 Brittingham J, Phiel C. Trzyna WC, et al. Identification of distinct molecular phenotypes in cultured gastrointestinal smooth muscle cells. Gastroenterology 1998; 115:605-17.

37 Martin JE, Benson M, Swash M, et al. Myofibroblasts in hollow visceral myopathy: the origin of gastrointestinal fibrosis? Gut 1993;34:999-1001.

38 Lindberg G, Glia A, Nyberg B, et al. Lymphocytic epithelioganglionitis-A new entity causing severe motility disorders of the gut. Gastroenterology 1999;116:G4476.

39 Schnappi NG, Smith VV, Milla PJ, et al. Eosinophilic myenteric ganglionitis is associated with functional intestinal obstruction. Gut 2003;52:752-5.

40 Grossi L, McHugh K, Collins SM. On the specificity of altered muscle function in experimental colitis in rats. Gastroenterology 1993;104:1049-56.

41 Weisbrodt NW, Lai M, Bowers RL, et al. Structural and molecular changes in intestinal smooth muscle induced by Trichinella spiralis infection. Am J Physiol 1994;266:G856-62.

\section{EDITOR'S QUIZ: GI SNAPSHOT}

\section{Bamboo trees in the stomach}

\section{Clinical presentation}

A 46 year old man presented with general fatigue and abdominal discomfort. He had a surgical history of ileal perforation at the age of 40 years. His family history was unremarkable. On examination, there was no tenderness on palpation of the abdomen with increased bowel sounds. Laboratory values were as follows: leucocytes $7.1 \times 10^{9} / 1$; haemoglobin $10.5 \mathrm{~g} / \mathrm{dl}$; and albumin $3.0 \mathrm{~g} / \mathrm{dl}$. Upper gastrointestinal endoscopy after spraying with a solution of indigo carmine dye showed swollen longitudinal folds transversed by linear furrows like "bamboo trees" on the lesser curvature of the gastric body (fig 1). Helicobacter pylori was negative.

\section{Question}

What characteristic structure will be shown in the biopsies? What is the diagnosis?

See page 1631 for answer

This case is submitted by:

A Hokama, F Kinjo, R Tomiyama, T Makishi, K Kobashigawa, T Sunagawa, Y Yonamine, Y Kugai, R Matayoshi, A Saito First Department of Internal Medicine, University of the Ryukyus, Okinawa,

Japan

Correspondence to: Dr A Hokama, First Department of Internal Medicine, University of the Ryukyus, 207 Uehara, Nishihara, Okinawa 903-0215, Japan; hokama-a@med.u-ryukyu.ac.jp

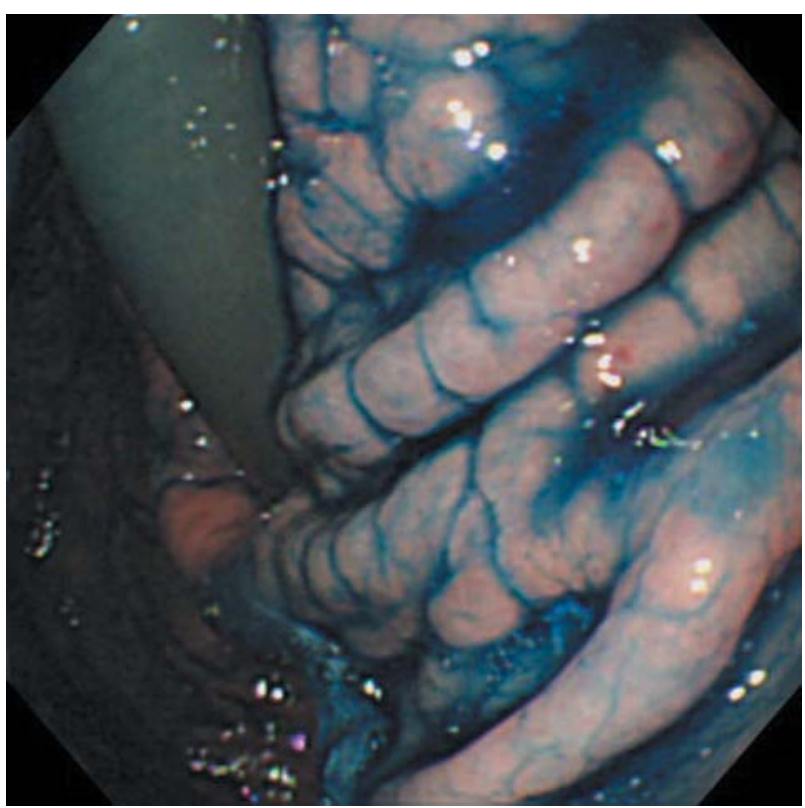

Figure 1 Upper gastrointestinal endoscopy.

doi: $10.1136 /$ gut.2003.038323 